\title{
Estramustine-Vinblastine Regimen
}

National Cancer Institute

\section{Source}

National Cancer Institute. Estramustine-Vinblastine Regimen. NCI Thesaurus. Code C64842.

A reg imen consisting of estramustine and vinblastine used for the treatment of hormone-refractory prostate cancer. 